\title{
Precise nitrogen depth profiling by high-resolution RBS in combination with angle-resolved XPS
}

\section{AUTHOR(S):}

Kimura, Kenji; Nakajima, Kaoru; Conard, Thierry; Vandervorst, Wilfried; Bergmaier, Andreas; Dollinger, Günther

\section{CITATION:}

Kimura, Kenji ... [et al]. Precise nitrogen depth profiling by high-resolution RBS in combination with angle-resolved XPS. Nuclear Instruments and Methods in Physics Research Section B: Beam Interactions with Materials and Atoms 2010, 268(11-12): 1960-1963

\section{ISSUE DATE:}

2010-06

URL:

http://hdl.handle.net/2433/126727

\section{RIGHT:}

(C) 2010 Elsevier B.V.; This is not the published version. Please cite only the published version.; この論文は出版社版でありません。引用の際に は出版社版をご確認ご利用ください。 


\title{
Precise nitrogen depth profiling by high-resolution RBS in combination with angle-resolved XPS
}

\author{
Kenji Kimura $^{\mathrm{a}^{*}}$, Kaoru Nakajima ${ }^{\mathrm{a}}$, Thierry Conard ${ }^{\mathrm{b}}$, Wilfried Vandervorst ${ }^{\mathrm{b}}$ \\ Andreas Bergmaier ${ }^{c}$, Günther Dollinger ${ }^{c}$
}

${ }^{a}$ Department of Micro Engineering, Kyoto University, Kyoto 606-8501, Japan,

${ }^{\mathrm{b}}$ IMEC, Kapeldreef 75, 3001 Leuven, Belgium,

${ }^{c}$ Institut für. Angewandte Physik und Messtechnik, Universität de Bundeswehr München, Werner-Heisenberg-Weg 39, D-85577 Neubiberg, Germany

Nitorgen depth profiling in a high-k gate stack structure, $\mathrm{SiON} / \mathrm{HfO}_{2} / \mathrm{SiON} / \mathrm{Si}(001)$ was performed by high-resolution Rutherford backscattering spectroscopy (HRBS) in combination with angle-resolved X-ray photoelectron spectroscopy (AR-XPS). The nitrogen depth profile is determined so that both the HRBS spectrum and the angular dependence of the XPS yield are reproduced. The obtained nitrogen profile is compared with the result of high-resolution elastic recoil detection (ERD) which is the most relaiable technique for depth profiling of light elements. The agreement between the result of the present combination analysis and that of high-resolution ERD is fairly good, showing that the present combination analysis is a promising method for the analysis of light elements.

PACS: 61.05.Np, 82.80.Pv

Keywords: High-resolution RBS, angle-resolved XPS, nitrogen depth profiling, combination analysis,

*Tel: +81-75-753-5253, Fax: +81-75-753-5253, E-mail: kimura@kues.kyoto-u.ac.jp 


\section{Introduction}

High-resolution Rutherford backscattering spectroscopy (HRBS) is becoming popular in various research fields and industries, especially in the micro-electronics industry. It allows non-destructive and quantitative analysis with a depth resolution better than $1 \mathrm{~nm}$ without any special sample pretreatment [1]. Although HRBS has many advantages, there are, of course, some drawbacks. For example, HRBS cannot analyze chemical states. In this respect, angle-resolved X-ray photoelectron spectroscopy (AR-XPS) can be a good complement to HRBS [2]. Recently, we developed a combination analysis of HRBS and AR-XPS which is able to perform precise depth profiling of chemical states [3]. In this method, both HRBS and AR-XPS measurements are performed with the same sample. The attenuation lengths $\lambda$ for the photoelectrons are determined so that the composition depth profiles obtained by HRBS reproduce the AR-XPS result. Depth profiles of chemical states are then derived so that both AR-XPS and HRBS results are reproduced.

In our previous paper, we assumed that the escape probabilities of photoelectrons emitted at a depth $x$ without inelastic scattering, which is called the depth distribution function (DDF), can be given by a simple exponential function

$$
P(x)=\exp \left(-\frac{x}{\lambda \cos \theta_{e}}\right),
$$

where $\theta_{\mathrm{e}}$ is the emission angle measured from the surface normal. When $\theta_{\mathrm{e}}$ exceeds $60^{\circ}$, however, this simple formula cannot be used due to the influence of elastic scattering, especially at a large $x$ [4]. As a result, the data observed at $\theta_{\mathrm{e}}>60^{\circ}$ cannot be used in AR-XPS analysis. The data at $\theta_{\mathrm{e}}>60^{\circ}$ are, however, crucial for accurate AR-XPS analysis [5]. Therefore, Eq. (1) should be improved to include the influence of elastic scattering for the precise AR-XPS analysis.

There is a database for calculation of DDFs, which was published by the National Institute of Standards and Technology (NIST) [6]. The database is based on a 
solution of the kinetic Boltzmann equation within the so-called transport approximation [7]. Accordingly, the effects of the elastic scattering on DDFs are taken into account. We have recently demonstrated that the DDFs calculated by the NIST database are reliable in a wide range of $\theta_{\mathrm{e}}$ up to $80^{\circ}$ by comparing the photoelectron yields calculated with the NIST database and the observed AR-XPS results [4]. If the NIST database is used instead of Eq. (1), AR-XPS analysis can be improved by including the important data observed at $\theta_{\mathrm{e}}>60^{\circ}$. By combining this improved AR-XPS with HRBS, much more precise depth profiling can be performed.

In this paper, the combination analysis of HRBS and the improved AR-XPS is applied to nitrogen depth profiling in $\mathrm{SiON} / \mathrm{HfO}_{2} / \mathrm{SiON} / \mathrm{Si}(001)$. Depth profiling of light elements is generally difficult for many surface analysis techniques including HRBS, especially light elements in heavy matrices. The most reliable technique for depth profiling of light elements is high-resolution elastic recoil detection (ERD), although it is one of the most expensive techniques requiring a rather big accelerator and a sophisticated spectrograph [8]. The nitrogen profile obtained by the present combination analysis is compared with the result of the high-resolution ERD to examine the accuracy of this combination analysis.

\section{Experimental}

A gate stack structure, $\mathrm{SiON} / \mathrm{HfO}_{2} / \mathrm{SiON} / \mathrm{Si}(001)$ was prepared by the following procedures. A 1.6-nm-thick SiON layer (referred to as interface SiON layer") was grown by a direct plasma nitridation on a cleaned $200 \mathrm{~mm} \mathrm{Si(001)} \mathrm{wafer.}$ A 2.5-nm-thick $\mathrm{HfO}_{2}$ layer was then deposited using atomic layer deposition (ALD) at $300^{\circ} \mathrm{C}$ on the $\mathrm{SiON} / \mathrm{Si}(001)$. On top of the $\mathrm{HfO}_{2}$ layer, a third layer of $\mathrm{Si}_{3} \mathrm{~N}_{4}$ (nominal thickness $\sim 0.5 \mathrm{~nm}$ ) was grown by chemical vapor deposition (CVD). This third layer is expected to be oxidized in the air (referred to as "surface SiON layer"). As a result, $\mathrm{SiON} / \mathrm{HfO}_{2} / \mathrm{SiON} / \mathrm{Si}(001)$ structure was formed. The thickness uniformity of the 
prepared layer was examined by ellipsometry. The measured standard deviation of the thickness was $\sim 2 \%$. The prepared Si wafer was divided into small pieces of $15 \times 15$ $\mathrm{mm}^{2}$, and these pieces were measured by HRBS and AR-XPS.

The details of the HRBS measurement were described elsewhere [1]. Just briefly, the sample was irradiated by a beam of $400 \mathrm{keV} \mathrm{He}^{+}$. The beam size and the typical beam current were $\sim 2 \times 2 \mathrm{~mm}^{2}$ and $\sim 50 \mathrm{nA}$, respectively. Energy spectra of $\mathrm{He}^{+}$ions scattered at $50^{\circ}$ were measured by a $90^{\circ}$ sector magnetic spectrometer which was equipped with a one-dimensional position-sensitive-detector (100 $\mathrm{mm}$ in length) consisting of micro-channel plates and a resistive anode. The energy window of the spectrometer was $25 \%$ of the central energy and the energy resolution was $0.1 \%$. The typical measurement time was 15 min. During the measurement of the "random" spectrum the sample was rotated around the axis of the surface normal to avoid the channeling/blocking effects. In addition to the random spectrum, the [111] axial channeling spectrum was also measured to reduce the $\mathrm{Si}$ substrate signal. The reduction of $\mathrm{Si}$ signal allows precise measurements of light elements (oxygen and nitrogen in the present case).

AR-XPS measurements were performed using a Theta Probe (Thermo Fisher Scientific Inc.) equipped with an $\mathrm{Al} \mathrm{K \alpha}$ source. This instrument allows us to collect AR-XPS data in parallel without tilting the sample. Photoelectron spectra of $\mathrm{Hf} 4 \mathrm{f}, \mathrm{Si}$ $2 \mathrm{p}, \mathrm{O} 1 \mathrm{~s}$ and $\mathrm{N} 1 \mathrm{~s}$ were measured at emission angles, $\theta_{\mathrm{e}}$, from $20^{\circ}$ to $80^{\circ}$ with respect to the surface normal. Shirley background subtraction and peak fitting were used when extracting photoelectron yields.

\section{Results and discussion}

\section{3-1. HRBS measurements}

The observed HRBS spectrum for $\mathrm{SiON} / \mathrm{HfO}_{2} / \mathrm{SiON} / \mathrm{Si}(001)$ is shown by open circles in Fig. 1 (the incident angle is $50.2^{\circ}$ with respect to the surface normal). The 
arrows show the energies of $\mathrm{He}$ ions scattered from constituent atoms ( $\mathrm{Hf}, \mathrm{Si}, \mathrm{O}$, and $\mathrm{N}$ ) located at the surface. A large peak seen at $\sim 390 \mathrm{keV}$ corresponds to $\mathrm{Hf}$ in the $\mathrm{HfO}_{2}$ layer and a small peak at $\sim 360 \mathrm{keV}$ corresponds to $\mathrm{Si}$ in the surface $\mathrm{SiON}$ layer. A plateau-like spectrum, which starts at $\sim 350 \mathrm{keV}$ and extends to lower energies, corresponds to the substrate $\mathrm{Si}$. The signals of oxygen and nitrogen overlap with this substrate Si spectrum. Due to this overlapping, precise analysis of these light elements is rather difficult. In order to reduce this difficulty, the incident $\mathrm{He}^{+}$beam was aligned to the [111] channeling direction.

The filled circles show the observed [111] channeling spectrum (only the energy region less than $370 \mathrm{keV}$ is shown because the $\mathrm{Hf}$ spectrum is essentially the same as the random spectrum). Compared with the random spectrum, the Si signal is significantly reduced and the oxygen peak is clearly seen at $\sim 330 \mathrm{keV}$. There is a small broad peak at $\sim 315 \mathrm{keV}$ which corresponds to nitrogen in the interface SiON layer. The nitrogen in the surface SiON layer, however, accidentally overlaps with the trailing edge of the oxygen peak. Nevertheless, the oxygen trailing edge is very smooth and no clear signature of the surface nitrogen is seen. This suggests that the concentration of nitrogen is negligibly small in the surface SiON layer. Therefore, the observed HRBS spectra were analyzed assuming that there is no nitrogen in the surface layer. The composition depth profiles were derived so that the profiles reproduce both the random and channeling spectra. The best fit results are shown by dashed lines in Fig. 1 and the obtained compositional depth profiles are shown in Fig. 2. Due to the above assumption the obtained nitrogen profile may have a large error while the profiles of other heavy elements should be much accurate. It should be noted that RBS can provide a depth scale in units of areal density $\left(\right.$ atoms $/ \mathrm{cm}^{2}$ ). This depth scale was converted into nm using the atomic density of $\mathrm{HfO}_{2}\left(8.3 \times 10^{22}\right.$ atoms $\left./ \mathrm{cm}^{3}\right)$ and the converted scale is indicated in the upper abscissa. Because the sample is not pure $\mathrm{HfO}_{2}$ this scale is just a guide. 


\section{3-2. AR-XPS measurements}

The same sample was measured by AR-XPS with Al Ka X-ray. The observed photoelectron yields were normalized by the cross sections and the effect of the asymmetric parameter was also corrected. The ratios of the corrected photoelectron yields, Hf4f/Si2p, O1s/Si2p and N1s/Si2p are shown as a function of $\theta_{\mathrm{e}}$ in Fig. 3.

\section{3-3. Combination analysis}

The DDFs for Hf4f, Si2p, O1s and N1s photoelectrons in $\mathrm{HfO}_{2}$ were calculated by the NIST database. Using these DDFs the $\theta_{\mathrm{e}}$-dependence of the photoelectron yields were calculated. In the calculation, the HRBS profiles shown in Fig. 2 were used. The calculated result (dashed lines) is compared with the observed one in Fig. 3. Although the calculated yield ratios, $\mathrm{Hf} 4 \mathrm{f} / \mathrm{Si} 2 \mathrm{p}$ and $\mathrm{O} 1 \mathrm{~s} / \mathrm{Si} 2 \mathrm{p}$, agree with the observed ones fairly well, there is a large discrepancy in N1s/Si2p. Recalling that the HRBS profiles other than nitrogen are accurate, the good agreement for Hf4f/Si2p and O1s/Si2p indicates that the DDFs calculated by the NIST database are reliable in the present case as was demonstrated in the previous study [4]. The large discrepancy for $\mathrm{N} 1 \mathrm{~s} / \mathrm{Si} 2 \mathrm{p}$ could be ascribed to the possible error in the nitrogen profiling in the present HRBS analysis. The calculated N1s/Si2p ratio is five times smaller than the observed result at $\theta_{\mathrm{e}} \sim 20^{\circ}$ and the discrepancy becomes larger with $\theta_{\mathrm{e}}$. This indicates that the assumption of no nitrogen in the surface $\mathrm{SiON}$ layer, which was used in the HRBS analysis, is not correct.

Now we will search the composition depth profiles so that both HRBS spectra and $\theta_{\mathrm{e}}$-dependences of the photoelectron yields can be reproduced. The profiles shown in Fig. 2 were used as the initial profiles in the fitting procedure and only oxygen and nitrogen profiles were allowed to be changed because the HRBS profiles of Hf and Si should be accurate. The best fit results are shown by solid lines in Figs 1 and 3 . 
Although the difference from the previous result is very small for the HRBS spectra (the result of the random spectrum is not shown because the difference is smaller than the thickness of the line), the improvement for AR-XPS is significant (the result for $\mathrm{Hf} 4 \mathrm{f} / \mathrm{Si} 2 \mathrm{p}$ is not shown because it is exactly the same as the previous result shown by a dashed line).

The obtained composition depth profiles are shown in Fig. 4. There is a small nitrogen peak at the surface. By integrating the nitrogen profile from 0 to $1.5 \times 10^{16}$ atoms $/ \mathrm{cm}^{2}$, the amount of nitrogen in the surface SiON layer is estimated to be $3.9 \times$ $10^{14}$ atoms $/ \mathrm{cm}^{2}$. This is much smaller than the nominal nitrogen density, $3 \times 10^{15}$ atoms $/ \mathrm{cm}^{2}$, calculated with the nominal thickness $(0.5 \mathrm{~nm})$ of the grown $\mathrm{Si}_{3} \mathrm{~N}_{4}$ layer (the density $3.4 \mathrm{~g} / \mathrm{cm}^{3}$ was used for $\mathrm{Si}_{3} \mathrm{~N}_{4}$ ). On the other hand, the amount of $\mathrm{Si}$ in the surface layer is estimated to be $1.6 \times 10^{15}$ atoms $/ \mathrm{cm}^{2}$ (estimated by integrating the $\mathrm{Si}$ profile from 0 to $1.5 \times 10^{16}$ atoms $/ \mathrm{cm}^{2}$ ), which is close to the nominal Si density, $2.2 \times$ $10^{15}$ atoms $/ \mathrm{cm}^{2}$ in the grown $\mathrm{Si}_{3} \mathrm{~N}_{4}$ layer. This suggests that the accuracy of the present combination analysis is not good for nitrogen depth profiling.

In order to estimate the accuracy of the present combination method, the sample was also measured by high-resolution ERD using a Q3D magnetic spectrograph at the Munich $14 \mathrm{MV}$ tandem accelerator [8]. The high-resolution ERD is one of the most reliable techniques for depth profiling of light elements, although it is a very sophisticated technique requiring a rather big facility. The obtained nitrogen profile is shown by a dotted line in Fig. 4, which is in good agreement with the HRBS profile. This clearly indicates that the present combination analysis is a promising method for the accurate analysis of light elements.

Both HRBS and high-resolution ERD indicate that the amount of the nitrogen in the surface $\mathrm{SiON}$ layer is almost one order of magnitude smaller than the designed value. This nitrogen reduction could be related to the growth mechanism of $\mathrm{Si}_{3} \mathrm{~N}_{4}$. When a $\mathrm{Si}_{3} \mathrm{~N}_{4}$ film is grown on a $\mathrm{Si}$ substrate the surface of the $\mathrm{Si}_{3} \mathrm{~N}_{4}$ film is covered by 
a thin Si layer of $\sim 1 \times 10^{15}$ atoms $/ \mathrm{cm}^{2}[9,10]$. This surface Si layer is oxidized upon exposure to air. The thickness of such $\mathrm{a} \mathrm{SiO}_{2}$ layer is $\sim 0.5 \mathrm{~nm}$, which is almost equal to the nominal thickness of the present $\mathrm{Si}_{3} \mathrm{~N}_{4}$ layer. If a similar surface Si layer was formed during the preparation of the present $\mathrm{Si}_{3} \mathrm{~N}_{4}$ layer on $\mathrm{HfO}_{2}$, the final structure of the sample should be $\mathrm{SiO}_{2} / \mathrm{Si}_{3} \mathrm{~N}_{4} / \mathrm{HfO}_{2} / \mathrm{SiON} / \mathrm{Si}(001)$. When the thickness of the $\mathrm{Si}_{3} \mathrm{~N}_{4}$ layer was estimated by ellipsometry, formation of such a $\mathrm{SiO}_{2}$ layer was not taken into account. Accordingly the thickness of the actual $\mathrm{Si}_{3} \mathrm{~N}_{4}$ layer should be much thinner than the nominal thickness in consistent with the present result. Looking at the nitrogen and oxygen profiles more closely (see Fig. 4), the surface nitrogen has a peak at $\sim 0.5 \mathrm{~nm}$ while oxygen has a peak at the surface. This confirms above explanation, a very thin $\mathrm{Si}_{3} \mathrm{~N}_{4}$ layer is covered by a $\mathrm{SiO}_{2}$ layer of $\sim 0.5 \mathrm{~nm}$.

\section{Conclusion}

A new method of the combination analysis of AR-XPS and HRBS was proposed for analysis of light elements. The method was applied to the analysis of the Hf-based gate stack structure, $\mathrm{SiON} / \mathrm{HfO}_{2} / \mathrm{SiON} / \mathrm{Si}(001)$. The nitrogen signal in the surface $\mathrm{SiON}$ layer accidentally overlaps with the trailing edge of the oxygen peak in the HRBS spectrum, which makes accurate nitrogen analysis difficult. In combination with AR-XPS, the nitrogen depth profile was derived from the measured HRBS spectra through spectrum simulation. The obtained nitrogen profile was in good agreement with the result of high-resolution ERD, showing that the proposed combination method is a promising method for the analysis of light elements.

\section{Acknowledgements}

This work was partly supported by SENTAN, JST. 


\section{References}

[1] K. Kimura, S. Joumori, Y. Oota, K. Nakajima and M. Suzuki, Nucl. Instr. and Methods B 219-220 (2004) 351.

[2] T. Hattori, T. Yoshida, T. Shiraishi, K. Takahashi, H. Nohira, S. Joumori, K. Nakajima, M. Suzuki, K. Kimura, I. Kashiwagi, C. Ohshima, S. Ohmi and H. Iwai, Microelectronic Engineering 72 (2004) 283.

[3] K. Kimura, K. Nakajima, M. Zhao, H. Nohira, T. Hattori, M. Kobata, E. Ikenaga, J. J. Kim, K. Kobayashi, T. Conard, W. Vandervorest, Surf. Interface Anal. 40 (2008) 423.

[4] K. Kimura, K. Nakajima, T. Conard, and W. Vandervorst, Appl. Phys. Lett. 91 (2007) 104106.

[5] C.J. Powell, W.S. Werner and W. Smekal, Appl. Phys. Lett. 89 (2006) 172101.

[6] C. J. Powell and A. Jablonski, NIST Electron Effective Attenuation Length Database, National Institute of Standards and Technology, version 1.0, Gaithersburg, MD, 2001.

[7] I. S. Tilinin, A. Jablonski, J. Zemek and S. Hucek, J. Electron Spectrosc. Relat. Phenom. 87, 127 (1997).

[8] G. Dollinger, A. Bergmaier, L. Goergens, P. Neumaier, W. Vandervorst and S. Jakschik, Nucl. Instr. and Methods B 219-220 (2004) 333.

[9] C. H. F. Peden, J. W. Rogers, Jr., N. D. Shinn, K. B. Kidd, and K. L. Tsang, Phys. Rev. B 47 (1993) 15622.

[10] T. Aratani, M. Higuchi, S. Sugawa, E. Okenaga, J. Ushio, H. Nohira, T. Suwa, A. Teramoto, T. Ohmi and T. Hattori, J. Appl. Phys. 104 (2008) 114112. 


\section{Figure captions}

Fig. 1 Random (open circles) and [111] channeling (solid circles) spectra of $\mathrm{SiON} / \mathrm{HfO}_{2} / \mathrm{SiON} / \mathrm{Si}(001)$. Only the energy region lower than $370 \mathrm{keV}$ is shown for the channeling spectrum. The arrows show the energies of He ions scattered from constituent atoms (Hf, Si, O, and N) located at the surface. The dashed line shows the best-fit result obtained by spectrum simulation without surface nitrogen. The solid line shows the best-fit result obtained by the combination analysis of HRBS and AR-XPS.

Fig. 2 Composition depth profiles of $\mathrm{SiON} / \mathrm{HfO}_{2} / \mathrm{SiON} / \mathrm{Si}(001)$ derived from the HRBS spectra shown in Fig. 1 assuming that there is no nitrogen in the surface SiON layer.

Fig. 3 Comparison between experimental (symbols) and calculated (lines) AR-XPS results for $\mathrm{SiON} / \mathrm{HfO}_{2} / \mathrm{SiON} / \mathrm{Si}$. The $\theta_{\mathrm{e}}$-dependence of the photoelectron yield ratios $\mathrm{Hf} 4 \mathrm{f} / \mathrm{Si} 2 \mathrm{p}, \mathrm{O} 1 \mathrm{~s} / \mathrm{Si} 2$ and $\mathrm{N} 1 \mathrm{~s} / \mathrm{Si} 2 \mathrm{p}$ are shown. The yield rations calculated with the HRBS depth profiles (Fig. 2) are shown by dashed lines. The solid lines show the best-fit result obtained by the combination analysis of HRBS and AR-XPS.

Fig. 4 Composition depth profiles of $\mathrm{SiON} / \mathrm{HfO}_{2} / \mathrm{SiON} / \mathrm{Si}$ obtained by the combination analysis of HRBS and AR-XPS. The nitrogen profile measured by high resolution ERD is also shown for comparison (dotted line). The agreement between the present result and high-resolution ERD is very good. 


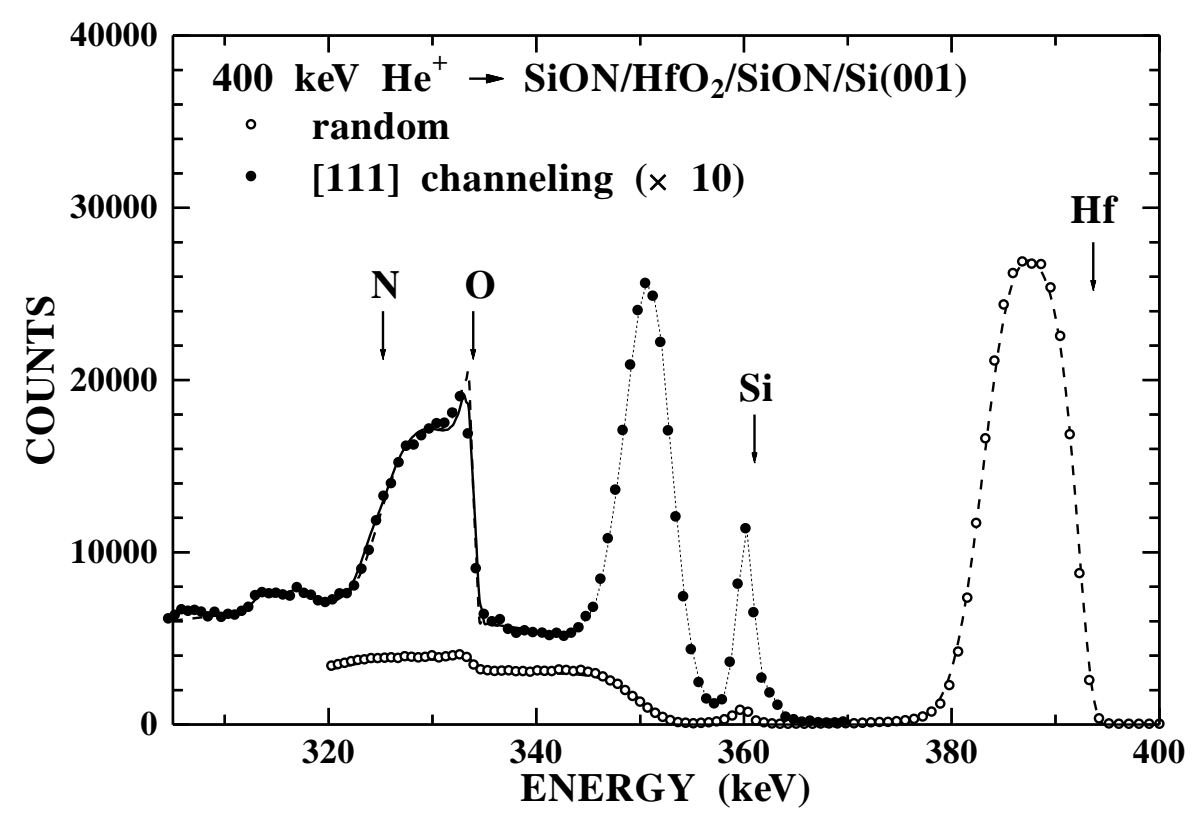

Fig. 1 K. Kimura et al

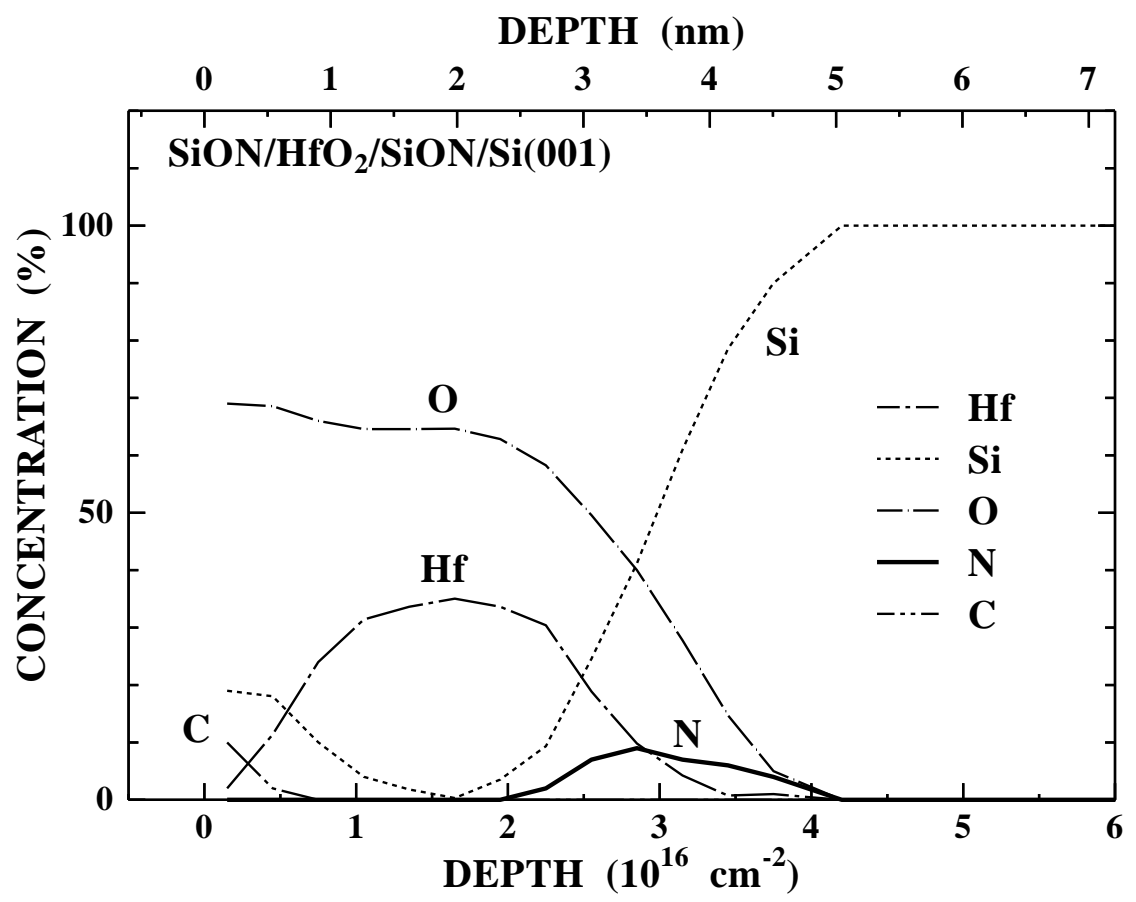

Fig. 2 K. Kimura et al 


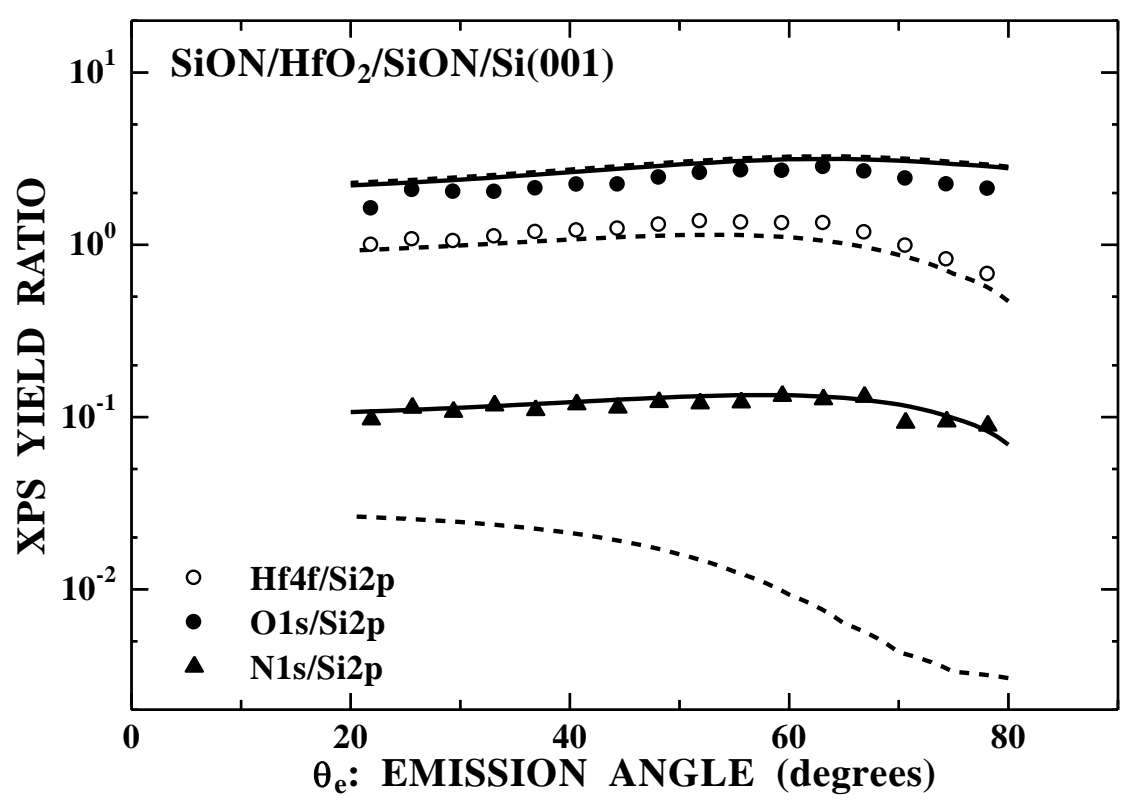

Fig. 3 K. Kimura et al

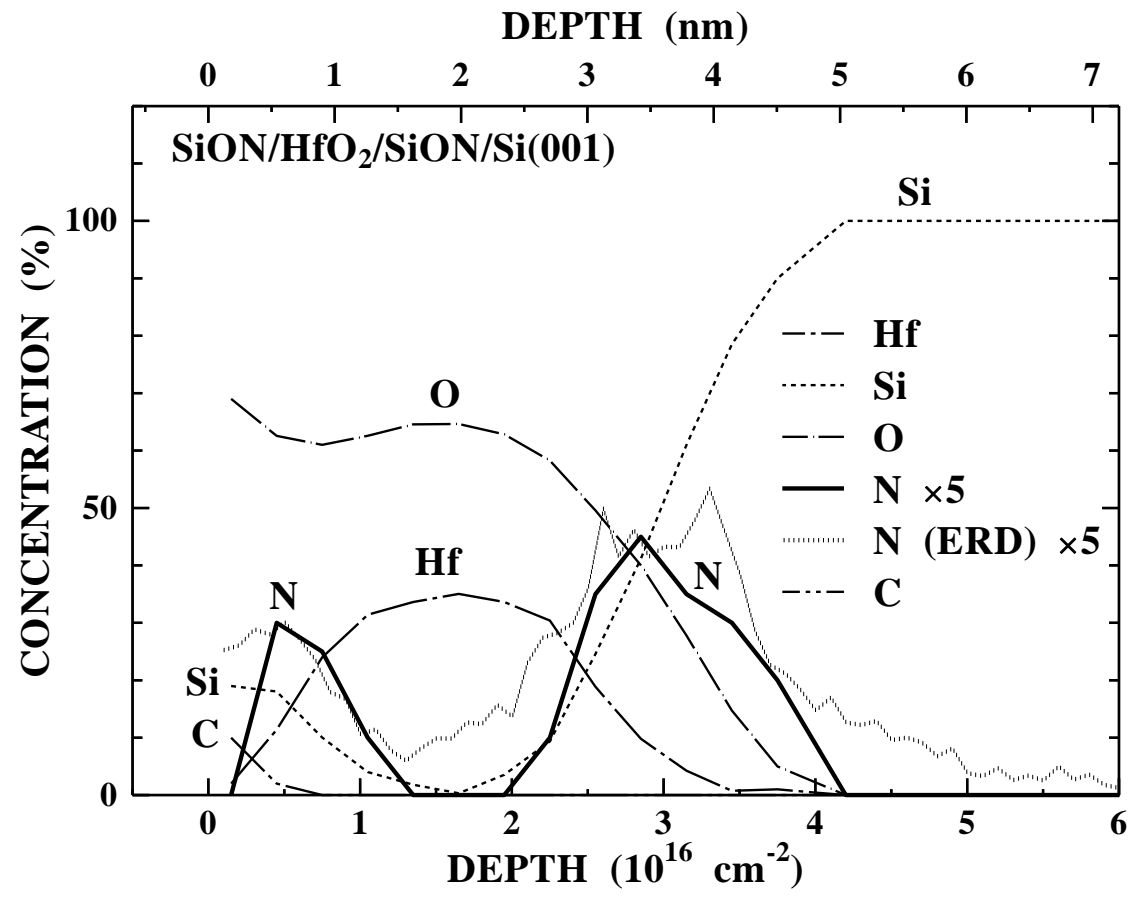

Fig. 4 K. Kimura et al 